1. Justify the inclusion of these extra-spinal manifestations in the set of classification criteria of axial spondyloarthritis

2. Justify specific investigations (mainly interview and physical exam ) of the patients at the time of the diagnosis

3. Justify a systematic monitoring of these different extra-spinal manifestations during the entire course of the disease

Disclosure of Interest: None declared

DOI: 10.1136/annrheumdis-2018-eular.7651

WEDNESDAY, 13 JUNE 2018

\section{Cancer and inflammation}

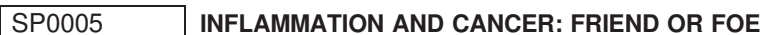

C.S. Roxburgh. Academic Unit of Surgery, University of Glasgow, Glasgow, UK

Cancer associated inflammation and the host immune response are key determinants of progression and outcome in solid malignancies. There is now persuasive evidence that inflammation is key to tumourigenesis via dna damage, stimulation of angiogenesis and proliferation, and inhibition of apoptosis. there have long been suspicions that dysregulated immune and inflammatory responses promote the progression and dissemination of established cancers and there is a heightened risk of cancer development in individuals with chronic inflammatory diseases. the tumour microenvironment is now viewed as a battleground for protumour and anti-tumour responses. We now see an increase array of novel therapeutic targets targeting innate and adaptive components of the immune response to cancer. ONCO-Immunology with immunotherapy are an established part of clinical practice differing from conventional anti-cancer treatments in that the strategies do not principally target cancer cells. This talk will focus on the evidence that inflammation influences malignant progression in gi malignancy discussing novel therapeutic strategies.

Disclosure of Interest: None declared

DOI: 10.1136/annrheumdis-2018-eular.7859

\section{WEDNESDAY, 13 JUNE 2018}

\section{Psychological distress and pain; not all in the mind.}

\section{SP0006 DISTRESS AND PAIN: INTEGRATED BRAIN PATHWAYS}

B. Sundermann. Institute of Clinical Radiology, University Hospital Muenster, Muenster, Germany

The focus of functional and multimodal neuroimaging studies has been switching from individual brain areas to larger interacting networks in the human brain. Regions within a network as well as different networks interact with each other and are usually involved in multiple brain functions such as perception, attention or cognitive control. In this presentation we will review the current knowledge on brain networks which are involved in the processing of painful stimuli or altered in patients suffering from chronic pain and how these share network correlates implicated in distress.

Disclosure of Interest: None declared

DOI: 10.1136/annrheumdis-2018-eular.7764

\section{SP0007 DEALING WITH PSYCHOLOGICAL DISTRESS TO OPTIMISE OUTCOMES FOR ARTHRITIS PAIN}

\section{R.J. Smeets. Rehabilitation Medicine, Maastricht University, Maastricht,} Netherlands

As clinicians we are often confronted with patients who suffer from arthritis pain. As most of us working in the field of arthritis, we are specifically trained to assess and treat medical causes in order to relieve pain. However, in daily practice we experience that many patients will develop chronic pain with huge problems in staying active and experiencing moderate to high levels of disability and lower level of quality of life.

In my opinion all clinicians should be able to identify psychosocial factors that contribute to the persistence of arthritis pain associated disability and loss of quality of life. Besides these patient relevant psychosocial factors, I will specifically focus on the important role clinician's beliefs/attitudes regarding pain in better treating and coaching patients with chronic arthritis pain.

Participants will learn how to look for these potentially contributing factors, how to diagnose the level of functioning of these patients and how to inform patients about their pain and role of important contributing psychosocial factors. I will discuss the way how you might better prescribe pain medication and other pain relieving treatments and finally how you can help them to stay active despite being in pain, reduce the risk of iatrogenic damage, and what type of treatments exist to address contributing psychosocial factors.

Finally, I will specifically elaborate on the Fear Avoidance Model as one of the currently most often used theoretical models to assess patients and on which basis a very successful treatment called graded exposure in vivo has been developed. Disclosure of Interest: None declared DOI: 10.1136/annrheumdis-2018-eular.7796

WEDNESDAY, 13 JUNE 2018

\section{Inflammation in the shadow of fibromyalgia}

\section{SP0008 $\quad$ A RA WITH PERSISTANT HIGH DISEASE ACTIVITY UNDER BIOLOGICAL TREATMENT: HOW DOES CHANGING DIAGNOSIS CHANGE MANAGEMENT AND OUTCOME?}

A.P. Trouvin. Pain Evaluation and Treatment Department, Hopital Cochin, Paris, France

We shall discuss the clinical case of a 45 years old woman. She has been diag nosed with rheumatoid arthritis, rheumatoid factor and ACPA are positive, acute phase reactants were elevated and no erosion was detected on the initial X-Rays. After a first line treatment with a conventional DMARD during 18 months, she experienced new flares with multiple synovitis and with elevated acute phase reactants. A TNF-inhibitor treatment was initiated. After 3 months, low disease activity was not reached hence biologic treatment was switched to abatacept After 3 months, the patient still complained of pain in her hands and feet but acute phase reactants normalised, DAS-28 score is 5,20 (TJC: 18 , SJC: 0, ESR $14 \mathrm{~mm} / \mathrm{h}$, VAS general health patient: 70/100). Question rose to change biological therapy once again. The case presentation will discuss how a possible fibromyalgia can, in this case, be confounding in the patient's clinical evaluation and therefore change the management of the patient.

We shall discuss the clinical case of a 45 years old woman. She has been diagnosed with rheumatoid arthritis, rheumatoid factor and ACPA are positive, acute phase reactants were elevated and no erosion was detected on the initial X-Rays After a first line treatment with a conventional DMARD during 18 months, she experienced new flares with multiple synovitis and with elevated acute phase reactants. A TNF-inhibitor treatment was initiated. After 3 months, low disease activity was not reached hence biologic treatment was switched to abatacept. After 3 months, the patient still complained of pain in her hands and feet but acute phase reactants normalised, DAS-28 score is 5,20 (TJC: 18 , SJC: 0, ESR $14 \mathrm{~mm} / \mathrm{h}$, VAS general health patient: 70/100). Question rose to change biological therapy once again. The case presentation will discuss how a possible fibromyalgia can, in this case, be confounding in the patient's clinical evaluation and therefore change the management of the patient.

Disclosure of Interest: None declared

DOI: 10.1136/annrheumdis-2018-eular.7722

\section{SP0009 FIBROMYALGIA ONSET OF SYSTEMIC LUPUS ERYTHEMATOSUS?}

\section{N. Hassan. Rheumatology, University Hospital of Wales, Cardiff, UK}

Systemic lupus erythematosus (SLE) and fibromyalgia often pose a diagnostic challenge to clinicians. The two conditions can coexist and the presence of one can complicate the diagnosis and management of the other. There is an increased prevalence of fibromyalgia amongst SLE patients compared to the general popu lation but the exact nature of this association is not yet fully understood. We describe a patient diagnosed with fibromyalgia who later went on to develop SLE. Disclosure of Interest: None declared DOI: 10.1136/annrheumdis-2018-eular.7747 